\title{
水热法合成白磷钙石
}

\author{
李国昌，王萍，刘长波 \\ (山东理工大学 材料科学与工程学院, 淄博 255049)
}

\begin{abstract}
摘 要: 以 $\mathrm{CaO} 、 \mathrm{Mg}\left(\mathrm{NO}_{3}\right)_{2}$ 和 $\mathrm{H}_{3} \mathrm{PO}_{4}$ 为原料, 通过水热法合成白磷钙石。采用 $\mathrm{X}$ 射线衍射 $(\mathrm{XRD})$ 、场发射扫描电子 显微镜(SEM)、热分析(TG-DTA)和 X 射线荧光光谱(XRF)进行了分析表征。结果表明, 随着初始溶液中 $\mathrm{Mg} /(\mathrm{Ca}+\mathrm{Mg})$

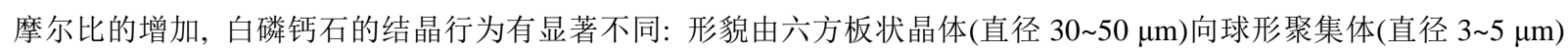
转变, 晶胞参数 $a$ 和 $c$ 值则逐渐减小。初始溶液中 $\mathrm{Mg} /(\mathrm{Ca}+\mathrm{Mg})$ 增加, 产物中的 $\mathrm{Mg} /(\mathrm{Ca}+\mathrm{Mg})$ 含量也逐渐增加, $\mathrm{Mg} /$ $(\mathrm{Ca}+\mathrm{Mg})$ 的最高值为 $13.6 \%$ 。在实验条件下, 白磷钻石的成核与结晶过程在 $2 \mathrm{~h}$ 之内基本完成。白磷钻石 $\mathrm{Ca}_{18} \mathrm{Mg}_{2} \mathrm{H}_{2}\left(\mathrm{PO}_{4}\right)_{14}$ 在 680 950 ${ }^{\circ} \mathrm{C}$ 之间脱去结构水, 转变为 $\mathrm{Ca}_{18} \mathrm{Mg}_{2}\left(\mathrm{PO}_{4}\right)_{12} \mathrm{P}_{2} \mathrm{O}_{7}$ 。

关 键 词: 水热法; 合成白磷钻石; 六方板状晶体; 球形聚集体
\end{abstract}

中图分类号: TB321 文献标识码: A

\section{Hydrothermal Synthesis of Whitlockite}

\author{
LI Guo-Chang, WANG Ping, LIU Chang-Bo
}

( Department of Material Science and Engineering, Shandong University of Technology, Zibo 255049, China)

\begin{abstract}
Whitlockite was firstly synthesized by a hydrothermal method using $\mathrm{CaO}, \mathrm{Mg}\left(\mathrm{NO}_{3}\right)_{2}$ and $\mathrm{H}_{3} \mathrm{PO}_{4}$ as starting materials and then characterized by X-ray diffraction, scanning electron morphology, thermal analysis, and X-ray fluorescence. The variation of molar ratio of $\mathrm{Mg} /(\mathrm{Ca}+\mathrm{Mg})$ in the precursor solution resulted in different crystallization behaviors of whitlockite during hydrothermal treatment. With the increase of the molar ratio of $\mathrm{Mg} /(\mathrm{Ca}+\mathrm{Mg}$ ), crystal morphologies of products varied from hexagonal plate (about 30-50 $\mu \mathrm{m}$ ) to spheres (about 3-5 $\mu \mathrm{m})$ and cell parameters of $a$ and $c$ values decreased gradually. Meanwhile, the content of $\mathrm{Mg} /(\mathrm{Ca}+\mathrm{Mg})$ in the products increased gradually and reached a high value of $13.6 \%$. It was found that the nucleation and growth of whitlockite were almost completed within $2 \mathrm{~h}$. $\mathrm{Ca}_{18} \mathrm{Mg}_{2} \mathrm{H}_{2}\left(\mathrm{PO}_{4}\right)_{14}$ was transformed to $\mathrm{Ca}_{18} \mathrm{Mg}_{2}\left(\mathrm{PO}_{4}\right)_{12} \mathrm{P}_{2} \mathrm{O}_{7}$ after calcination at temperature ranging from $680^{\circ} \mathrm{C}$ to $950^{\circ} \mathrm{C}$ due to the dehydration reaction.
\end{abstract}

Key words: hydrothermal method; synthetic whitlockite; hexagonal plate-like crystal; microsphere

磷酸钙(CaPs) 作为有吸引力的生物相容性材料, 一直受到研究者的关注 ${ }^{[1-6]}$ 。白磷钙石(Whitlockite, $\left.\mathrm{WH}, \mathrm{Ca}_{18} \mathrm{Mg}_{2} \mathrm{H}_{2}\left(\mathrm{PO}_{4}\right)_{14}\right)$ 是一种罕见的磷酸盐矿物, 存在于生物系统 ${ }^{[7]}$ 、月岩和陨石样品中 ${ }^{[8]}$ 。最新研 究表明, WH是人类骨骼中含量居第二位的重要矿 物, 在骨骼中的含量约为 $20 \mathrm{wt} \%$, 特别是在承重骨 骼中存在 ${ }^{[9]}$ 。与羟基磷灰石 $(\mathrm{HA})$ 不同, WH在酸性条 件下相对稳定。此外, 在青少年的骨骼中WH的含量
较高, 表明其可能积极参与骨的重塑过程 ${ }^{[10]}$ 。

$\mathrm{WH}\left(\mathrm{Ca}_{18} \mathrm{Mg}_{2} \mathrm{H}_{2}\left(\mathrm{PO}_{4}\right)_{14}\right)$ 与 $\beta-\mathrm{TCP}\left(\mathrm{Ca}_{18} \mathrm{Mg}_{2} \mathrm{Ca}\left(\mathrm{PO}_{4}\right)_{14}\right.$, $\left.\beta-\mathrm{Ca}_{3}\left(\mathrm{PO}_{4}\right)_{2}\right)$ 是一个固溶体系列的两个端元组分, 两 者具有相同的空间群 R3c。但是 $\beta$-TCP 是一种高温 相, 它不能从水溶液中直接沉淀出来, 它的最常见 制备方法是: 先采用化学沉淀或固态反应法获得钻 缺乏羟基磷灰石 $(\mathrm{CDHA})$ 或者 $\mathrm{Ca} / \mathrm{P} \leqslant 1.5$ 的无定形磷 酸钙粉末, 在粉末制备过程中加入掺杂离子, 再通 过焙烧(低于 $1120^{\circ} \mathrm{C}$ )粉末, 最终获得多晶 $\beta-\mathrm{TCP}$ 。 
在上述方法中, 高温焙烧是获得纯相以及离子替代 $\beta$-TCP 不可缺少的途径 ${ }^{[1-15]}$ 。

$\beta$-TCP 必须在高温下制备, WH 却可以从水溶 液中直接沉淀出来, 而目前关于沉淀 WH 的制备方 法和应用性能的研究文献很少 ${ }^{[9,16-17]}$ 。本文报道一种 合成 WH 的方法, 并研究了反应溶液中 $\mathrm{Mg} /(\mathrm{Mg}+\mathrm{Ca})$ 摩尔比、反应时间对 WH 的结构、成分和形貌的影 响以及高温条件下的成分与结构变化等。

\section{1 实验方法}

按照 $(\mathrm{Ca}+\mathrm{Mg})$ 摩尔浓度为 $0.1 \mathrm{~mol} / \mathrm{L}, \mathrm{Mg} /(\mathrm{Ca}+\mathrm{Mg})$ 摩尔比为 $0 \sim 0.5$, 将 $\mathrm{CaO}$ 和 $\mathrm{Mg}\left(\mathrm{NO}_{3}\right)_{2}$ 加去离子水, 摚 拌形成悬浮液 (1)。将 $\mathrm{H}_{3} \mathrm{PO}_{4}$ 配成所需浓度的溶液 (2)。 按 $(\mathrm{Ca}+\mathrm{Mg}) / \mathrm{P}$ (摩尔比 $)=1.67$, 摚拌下将溶液 (2)加入 到悬浮液 (1)中。向混合液中滴入硝酸, 待沉淀溶解, 溶液澄清后, 加入乙酰胺作为沉淀剂。将溶液转入 高压釜中, 在 $180^{\circ} \mathrm{C}$ 下水热反应 $2 \sim 6 \mathrm{~h}$ 。自然冷却后, 将所得沉淀洗涤、过滤, $120^{\circ} \mathrm{C}$ 干燥。将初始溶液中 $\mathrm{Mg} /(\mathrm{Ca}+\mathrm{Mg})$ 摩尔比为 $0 、 0.1 、 0.2 、 0.3 、 0.4 、 0.5$ 的 样品, 分别用 $\mathrm{Mg}_{0} 、 \mathrm{Mg}_{0.1} 、 \mathrm{Mg}_{0.2} 、 \mathrm{Mg}_{0.3} 、 \mathrm{Mg}_{0.4}$ 、 $\mathrm{Mg}_{0.5}$ 表示。

\section{2 样品表征}

采用德国布鲁克 D8-ADVANCE 型多晶 $\mathrm{X}$ 射线 衍射仪进行结构分析, 扫描范围 $2 \theta=5^{\circ} \sim 60^{\circ}$, 步长 $0.02^{\circ}$, 扫描速度 $2 \% \mathrm{~min}$, 电压 $40 \mathrm{kV}$, 电流 $35 \mathrm{~mA}$ 。 用 MDI Jade6.1 软件对衍射数据进行物相检索和晶 胞参数计算。采用日本理学 ZSX-100e 型 X 射线荧 光光谱仪分析产物的化学组成。采用德国 NETZSCH STA 449 C 型同步热分析质谱仪进行差 热分析, 温度范围 $27 \sim 1200^{\circ} \mathrm{C}$, 升温速度 $10^{\circ} \mathrm{C} / \mathrm{min}$ 。 采用荷兰 FEI 公司 Sirion 200 型场发射扫描电子显 微镜观察产物的形貌和尺寸。

\section{3 结果与讨论}

\section{1 初始溶液中 $\mathrm{Mg} /(\mathrm{Ca}+\mathrm{Mg})$ 摩尔比}

\subsubsection{Mg/(Ca+Mg)对产物的物相组成的影响}

$\mathrm{Mg}_{0}$ 是结晶良好的羟基磷灰石(图谱没有示出)。 $\mathrm{Mg}_{0.1} \sim \mathrm{Mg}_{0.5}$ 的 5 个样品的 XRD 图谱(图 1)的主要衍 射峰均与标准 WH(JCPDS ${ }^{\#}$ 70-2064)吻合, 表明 5 个 样品均由纯 WH 相组成。可见当溶液中存在合适浓
度的 $\mathrm{Mg}^{2+}$ 且初始 $\mathrm{pH}$ 较低(2.5 3)时, 更有利于 $\mathrm{WH}$ 的成核与生长。随着初始溶液中 $\mathrm{Mg}$ 含量增大, 样 品的主要衍射峰向高角度偏移, 其中 $\mathrm{Mg}_{0.3}$ 与标准 图谱 $\left((0210)\right.$ 面的衍射峰对应于 $\left.2 \theta=31.280^{\circ}\right)$ 最为接 近。从 MDI jade6.1 软件精修的晶胞参数可以看出 (表 1), 随着溶液中 $\mathrm{Mg}$ 含量的增加, $\mathrm{WH}$ 的晶胞参数 $a$ 和 $c$ 值都逐渐减小, 这是由于 $\mathrm{Mg}^{2+}$ 离子的半径 $(0.072 \mathrm{~nm})$ 值小于 $\mathrm{Ca}^{2+}$ 离子 $(0.10 \mathrm{~nm})$ 半径, 所以随 着 $\mathrm{WH}$ 结构中 $\mathrm{Mg}^{2+}$ 离子含量的增加, 晶胞参数的逐 渐减小。

\subsection{2 $\mathrm{Mg} /(\mathrm{Ca}+\mathrm{Mg})$ 对产物的化学成分的影响}

根据 XRF 分析结果计算的晶体化学组成列于 表 2, 从表中可以看出，随着反应溶液中 $\mathrm{Mg} /(\mathrm{Ca}+\mathrm{Mg})$ $\left(\mathrm{Mg}_{\mathrm{L}}\right)$ 的增加, 固体样品中的镁含量 $\mathrm{Mg} /(\mathrm{Ca}+\mathrm{Mg})$ $\left(\mathrm{Mg}_{\mathrm{S}}\right)$ 也逐渐增加, 但 $\mathrm{Mg}_{\mathrm{S}} / \mathrm{Mg}_{\mathrm{L}}$ 却不断降低。WH 的 理论 $\mathrm{Mg} /(\mathrm{Ca}+\mathrm{Mg})$ 为 $0.1,(\mathrm{Ca}+\mathrm{Mg}) / \mathrm{P}=1.5$ 。根据目前 的实验结果, 只有 $\mathrm{Mg}_{\mathrm{L}}$ 达到 0.3 的较高浓度时才能 形成接近化学计量的 $\mathrm{WH}$ 。反应溶液中 $\mathrm{Mg} /(\mathrm{Ca}+\mathrm{Mg})$ 为 0.5 时, 白磷钻石中 $\mathrm{Mg} /(\mathrm{Ca}+\mathrm{Mg})$ 达到 0.136 。

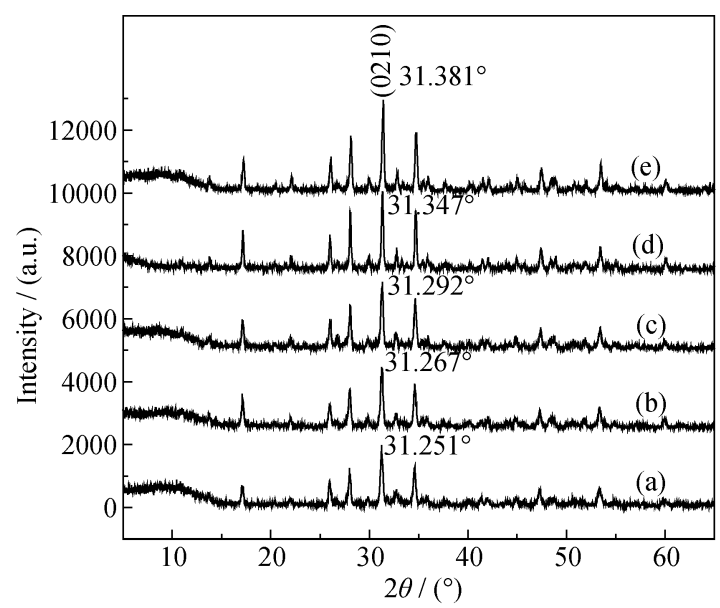

图 1 不同 $\mathrm{Mg} /(\mathrm{Ca}+\mathrm{Mg})$ 样品的 XRD 图谱

Fig. 1 XRD patterns of different $\mathrm{Mg} /(\mathrm{Ca}+\mathrm{Mg})$ samples $\left(180^{\circ} \mathrm{C}\right.$, $6 \mathrm{~h})$

(a) $\mathrm{Mg}_{0.1}$; (b) $\mathrm{Mg}_{0.2}$; (c) $\mathrm{Mg}_{0.3}$; (d) $\mathrm{Mg}_{0.4}$; (e) $\mathrm{Mg}_{0.5}$

表 1 根据 XRD 数据精修的晶胞参数 Table 1 Unit cell parameters of samples

\begin{tabular}{ccc}
\hline \multirow{2}{*}{ Sample } & \multicolumn{2}{c}{ Unit cell parameters } \\
\cline { 2 - 3 } & $a / \mathrm{nm}$ & $c / \mathrm{nm}$ \\
\hline $\mathrm{Mg}_{0.1}$ & 1.03705 & 3.71939 \\
$\mathrm{Mg}_{0.2}$ & 1.03707 & 3.71234 \\
$\mathrm{Mg}_{0.3}$ & 1.03542 & 3.71068 \\
$\mathrm{Mg}_{0.4}$ & 1.03431 & 3.70353 \\
$\mathrm{Mg}_{0.5}$ & 1.03412 & 3.70203 \\
$\mathrm{PDF} \#$ 70-2064 & 1.03500 & 3.70850 \\
\hline
\end{tabular}


表 2 不同 $\mathrm{Mg} /(\mathrm{Ca}+\mathrm{Mg})$ 样品的化学成分

Table 2 Chemical constituents of different $\mathrm{Mg} /(\mathrm{Ca}+\mathrm{Mg})$ samples

\begin{tabular}{lcccccc}
\hline \multirow{2}{*}{$\mathrm{Mg}_{\mathrm{L}}$} & \multicolumn{3}{c}{ Chemical constituents/wt\% } & \multicolumn{3}{c}{ Molar ratio } \\
\cline { 2 - 7 } & $\mathrm{CaO}$ & $\mathrm{P}_{2} \mathrm{O}_{5}$ & $\mathrm{MgO}$ & $\mathrm{Mg}$ & $\mathrm{Mg}_{\mathrm{L}} / \mathrm{Mg}_{\mathrm{S}}$ & $(\mathrm{Ca}+\mathrm{Mg}) / \mathrm{P}$ \\
\hline $\mathrm{Mg}_{0.1}$ & 53.95 & 43.85 & 1.85 & 0.041 & 0.41 & 1.62 \\
$\mathrm{Mg}_{0.2}$ & 50.95 & 45.64 & 3.16 & 0.076 & 0.38 & 1.59 \\
$\mathrm{Mg}_{0.3}$ & 49.34 & 46.04 & 4.21 & 0.102 & 0.34 & 1.51 \\
$\mathrm{Mg}_{0.4}$ & 48.54 & 46.34 & 4.89 & 0.119 & 0.30 & 1.51 \\
$\mathrm{Mg}_{0.5}$ & 46.74 & 46.8 & 6.03 & 0.136 & 0.27 & 1.49 \\
\hline
\end{tabular}

对于 $\mathrm{Mg}_{0.1}$ 和 $\mathrm{Mg}_{0.2}$, 产物的 $(\mathrm{Ca}+\mathrm{Mg}) / \mathrm{P}$ (摩尔比) 高于 1.5 , 可能与其含有微量的 HA 有关。HA 是在 水热反应后期溶液 $\mathrm{pH}$ 升高时形成的。

\subsubsection{Mg/(Ca+Mg)对产物形貌的影响}

样品 $\mathrm{Mg}_{0} \sim \mathrm{Mg}_{0.5}$ 的 SEM 形貌示于图 2, $\mathrm{Mg}_{0}$ 为结 晶良好的 $\mathrm{HA}$ 纤维(图 2(a)); $\mathrm{Mg}_{0.1}$ 和 $\mathrm{Mg}_{0.2}$ 主要为六方 板状 WH 晶体; $\mathrm{Mg}_{0.3}$ 中除了六方板状晶体之外, 出现 了较多球形聚集体; $\mathrm{Mg}_{0.4}$ 和 $\mathrm{Mg}_{0.5}$ 全部由球形聚集体 组成。此外, 随着 $\mathrm{Mg}^{2+}$ 浓度的增加, $\mathrm{WH}$ 单晶体和球 形聚集体的尺寸都在逐渐减小。文献研究显示, 镁离 子替代可使沉淀 HAP 的结晶度降低 ${ }^{[18]}$ 。本研究显示, 镁离子替代对沉淀 WH 也有同样的影响, 导致六方板 状晶体和球形聚集体的尺寸随 $\mathrm{Mg} /(\mathrm{Ca}+\mathrm{Mg})$ 的增加而 减小，其机制尚需进一步研究。
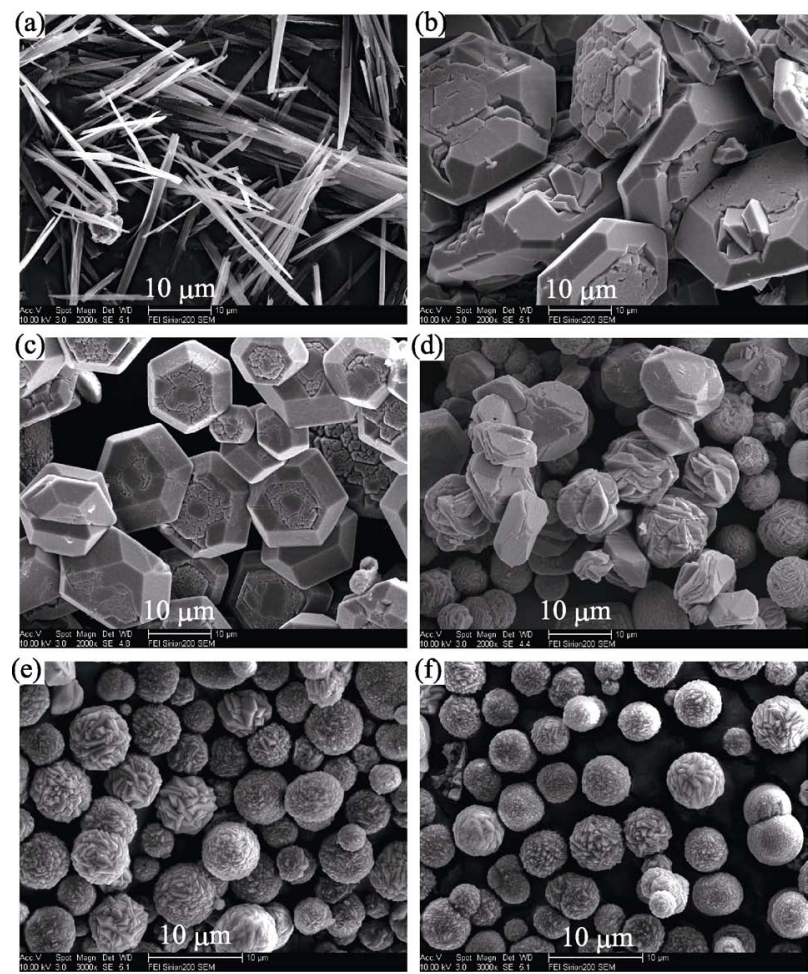

图 2 不同 $\mathrm{Mg} /(\mathrm{Ca}+\mathrm{Mg})$ 样品的 SEM 照片

Fig. 2 SEM images of different $\mathrm{Mg} /(\mathrm{Ca}+\mathrm{Mg})$ samples $\left(180^{\circ} \mathrm{C}\right.$, $6 \mathrm{~h})$

(a) $\mathrm{Mg}_{0}(\mathrm{HA})$; (b) $\mathrm{Mg}_{0.1}$; (c) $\mathrm{Mg}_{0.2}$; (d) $\mathrm{Mg}_{0.3}$; (e) $\mathrm{Mg}_{0.4}$; (f) $\mathrm{Mg}_{0.5}$

\section{2 水热反应时间}

\subsection{1 水热时间对物相组成的影响}

水热时间为 90 和 $100 \mathrm{~min}$ 时，反应溶液中没有 沉淀生成。反应时间增加到 $2 \mathrm{~h}$ 时, 溶液中出现 $\mathrm{WH}$ 沉淀(图 3(a)), 继续延长反应时间至 3、4、6 h(图 3(b), (c), (d)), 产物的相组成基本没有变化。

\subsection{2 水热时间对 WH 形貌的影响}

不同反应时间得到的 $\mathrm{Mg}_{0.3}$ 样品的 SEM 形貌示 于图 4。对于 $\mathrm{Mg}_{0.3}, \mathrm{WH}$ 呈单晶体与球形聚集体。 从图 4 可以看出, 反应 2 6 h 得到的样品, 随着反应 时间的增加, 单晶体与球形聚集体的尺寸都在增加, 且尺寸趋于一致。反应 $10 \mathrm{~h}$ 样品的尺寸与反应 $6 \mathrm{~h}$ 样品相比变化不大。

由于在反应最初 90 和 $100 \mathrm{~min}$ 时无沉淀生成, 可以认为, 反应 $100 \mathrm{~min}$ 后 WH才开始成核, 结晶与 熟化过程在反应 $6 \mathrm{~h}$ 之内基本结束。按照奥斯特瓦 尔德熟化机理, 随着反应时间的延长, 溶液浓度降 低, 小晶体会溶解, 并重新沉积在热力学稳定的大 晶体表面, 使晶体不断长大。

\section{3 高温下 WH 的成分与结构变化}

为了确认合成样品中结构水的存在, 从室温 $\sim 1200^{\circ} \mathrm{C}$ 对产物进行热分析。样品 $\mathrm{Mg}_{0.1} \sim \mathrm{Mg}_{0.5}$ 的热 分析结果基本相同, 图 5 仅示出了 $\mathrm{Mg}_{0.3}$ 样品的 $\mathrm{TG}$ 和 DTA 曲线。从 TG 曲线可以看出, 从室温升至 $680^{\circ} \mathrm{C}$ 的质量损失约为 $1.26 \%$, 这与 $\mathrm{WH}$ 中的吸附水 脱附有关; 从 $680^{\circ} \mathrm{C}$ 升至 $950^{\circ} \mathrm{C}$, 质量损失为 $0.85 \%$, 与 $\mathrm{WH}$ 结构水脱附有关, 这是存在结构水的直接证 据。WH 的晶体化学式为 $\mathrm{Ca}_{18} \mathrm{Mg}_{2} \mathrm{H}_{2}\left(\mathrm{PO}_{4}\right)_{14}$ (或写成 $\left.\left(\mathrm{Ca}_{18} \mathrm{Mg}_{2}\right)\left(\mathrm{PO}_{4}\right)_{12}\left(\mathrm{PO}_{3} \mathrm{OH}\right)_{2}\right)$, 当失去结构中水转变 为 $\mathrm{Ca}_{18} \mathrm{Mg}_{2}\left(\mathrm{PO}_{4}\right)_{12} \mathrm{P}_{2} \mathrm{O}_{7}$ 时, 理论质量损失为 $0.86 \%$, 热分析结果与理论计算一致。此外, DTA 曲线为一 宽的吸热峰, 峰值位于 $874^{\circ} \mathrm{C}$, 与上述脱水反应对 应。DTA 曲线在 $1158^{\circ} \mathrm{C}$ 的放热峰, 代表新相的形成。

根据热分析结果, 将沉淀 $\mathrm{WH}$ 分别在 $950^{\circ} \mathrm{C}$ 和 $1200^{\circ} \mathrm{C}$ 焙烧 $2 \mathrm{~h}$, 升温速度 $2^{\circ} \mathrm{C} / \mathrm{min}$ 。焙烧后样品的 XRD 分析结果示于图 6, 可以看出, 焙烧样品与沉 
淀 WH 的 XRD 图谱基本相同，只是衍射峰的强度有 所增强, 位置略向高角度偏移, 晶胞参数 $a$ 和 $c$ 值有 所减小(表 3)。此外, 焙烧样品中均观察到 $\mathrm{Ca}_{2} \mathrm{P}_{2} \mathrm{O}_{7}$ (JCPDS 20-24)的衍射峰。

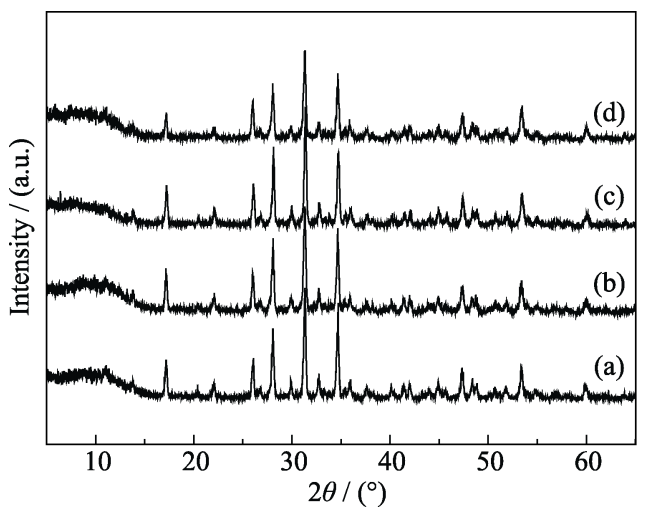

图 3 不同反应时间得到的样品的 XRD 图谱

Fig. 3 XRD patterns of samples reacted for different time $\left(\mathrm{Mg}_{0.3}, 180^{\circ} \mathrm{C}\right)$

(a) $2 \mathrm{~h}$; (b) $3 \mathrm{~h}$; (c) $6 \mathrm{~h}$; (d) $10 \mathrm{~h}$
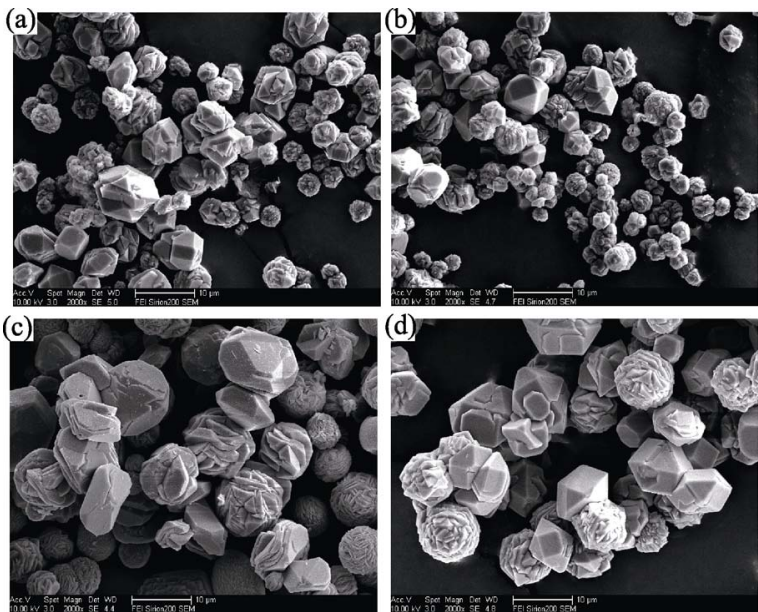

图 4 不同反应时间得到的 $\mathrm{Mg}_{0.3}$ 样品的 SEM 照片

Fig. 4 SEM images of samples reacted for different time $\left(\mathrm{Mg}_{0.3}, 180^{\circ} \mathrm{C}\right)$

(a) $2 \mathrm{~h}$; (b) $3 \mathrm{~h}$; (c) $6 \mathrm{~h}$; (d) $10 \mathrm{~h}$

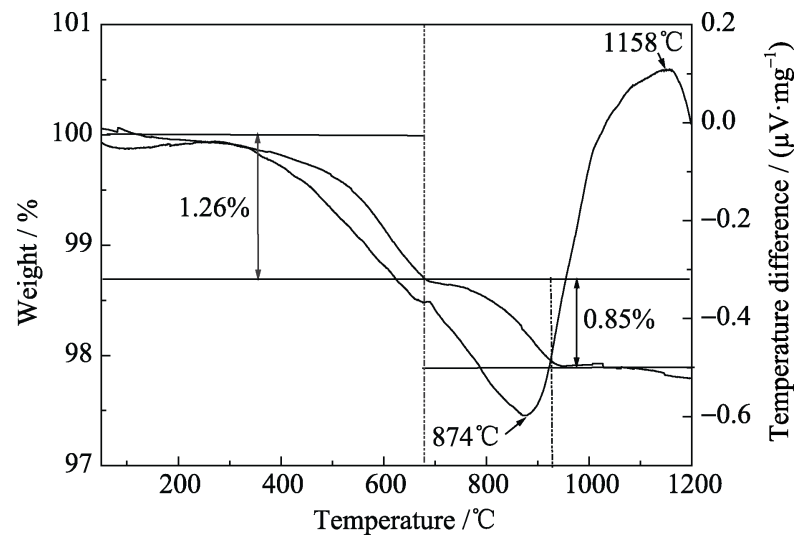

图 $5 \mathrm{Mg}_{0.3}$ 样品的 TG-DTA 曲线

Fig. 5 TG and DTA curves of sample $\mathrm{Mg}_{0.3}$

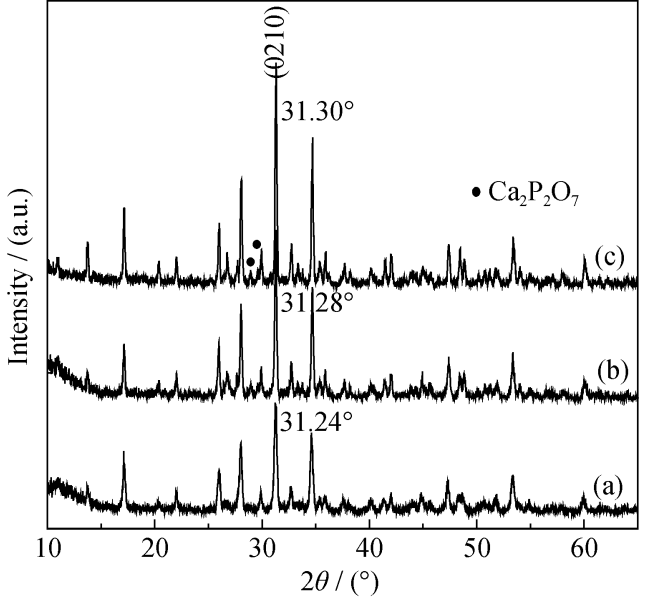

图 6 焙烧样品的 XRD 图谱

Fig. 6 XRD patterns of the calcined samples

(a) As-prepared sample $\left(\mathrm{Ca}_{18} \mathrm{Mg}_{2} \mathrm{H}_{2}\left(\mathrm{PO}_{4}\right)_{14}\right)$; (b) Calcined at $950^{\circ} \mathrm{C}$ $\left(\mathrm{Ca}_{18} \mathrm{Mg}_{2}\left(\mathrm{PO}_{4}\right)_{12} \mathrm{P}_{2} \mathrm{O}_{7}\right), 2 \mathrm{~h}$; (c) Calcined at $1200^{\circ} \mathrm{C}, 2 \mathrm{~h}\left(\mathrm{Ca}_{18} \mathrm{Mg}_{2}\left(\mathrm{PO}_{4}\right)_{12} \mathrm{P}_{2} \mathrm{O}_{7}\right)$

结合 DTA 和 TG 分析, 可以看出, 焙烧使 WH 结构 脱水, 由 $\mathrm{Ca}_{18} \mathrm{Mg}_{2} \mathrm{H}_{2}\left(\mathrm{PO}_{4}\right)_{14}$ 转变为 $\mathrm{Ca}_{18} \mathrm{Mg}_{2}\left(\mathrm{PO}_{4}\right)_{12} \mathrm{P}_{2} \mathrm{O}_{7}$, 这种转变导致 WH 的质量损失 $(0.85 \%$, 图 5)和晶胞参 数的减小, 而晶体结构型式没有发生根本改变。此外, $\mathrm{Ca}_{2} \mathrm{P}_{2} \mathrm{O}_{7}$ 的出现, 也与上述转变有关。

表 3 焙烧样品的晶胞参数

Table 3 Unit cell parameters of calcined samples

\begin{tabular}{cccc}
\hline \multirow{2}{*}{$\begin{array}{c}\text { Calcination } \\
\text { temperature }\end{array}$} & Chemical formula & \multicolumn{2}{c}{$\begin{array}{c}\text { Unit cell } \\
\text { parameters }\end{array}$} \\
\cline { 3 - 4 } & & $a / \mathrm{nm}$ & $c / \mathrm{nm}$ \\
\hline As-prepared sample & $\mathrm{Ca}_{18} \mathrm{Mg}_{2} \mathrm{H}_{2}\left(\mathrm{PO}_{4}\right)_{14}$ & 1.03707 & 3.71234 \\
$950^{\circ} \mathrm{C}$ & $\mathrm{Ca}_{18} \mathrm{Mg}_{2}\left(\mathrm{PO}_{4}\right)_{12} \mathrm{P}_{2} \mathrm{O}_{7}$ & 1.03472 & 3.71176 \\
$1200^{\circ} \mathrm{C}$ & $\mathrm{Ca}_{18} \mathrm{Mg}_{2}\left(\mathrm{PO}_{4}\right)_{12} \mathrm{P}_{2} \mathrm{O}_{7}$ & 1.03412 & 3.71094 \\
\hline
\end{tabular}

\section{4 结论}

采用水热法 $180^{\circ} \mathrm{C}$ 成功合成了白磷钙石 $(\mathrm{WH})$ 。 初始溶液中的 $\mathrm{Mg} /(\mathrm{Ca}+\mathrm{Mg})$ 摩尔比对 $\mathrm{WH}$ 晶体形貌 和晶胞参数影响较大, 即随着 $\mathrm{Mg} /(\mathrm{Ca}+\mathrm{Mg})$ 摩尔比 的增加, WH 形貌由六方板状晶体变为球形聚集体, 晶胞参数 $a$ 和 $c$ 值都逐渐减小; 随着水热反应时间 的延长, $\mathrm{WH}$ 晶体尺寸增加; 热处理表明, $950^{\circ} \mathrm{C}$ 开 始, 白磷钙石 $\left(\mathrm{Ca}_{18} \mathrm{Mg}_{2} \mathrm{H}_{2}\left(\mathrm{PO}_{4}\right)_{14}\right)$ 脱去结构水, 转变 为 $\mathrm{Ca}_{18} \mathrm{Mg}_{2}\left(\mathrm{PO}_{4}\right)_{12} \mathrm{P}_{2} \mathrm{O}_{7}$ 。

参考文献:

[1] YASHIMA M, SAKAI A, KAMIYAMA T, et al. Crystal structure analysis of $\beta$-tricalcium phosphate $\mathrm{Ca}_{3}\left(\mathrm{PO}_{4}\right)_{2}$ by neutron powder diffraction. Journal of Solid State Chemistry, 2003, 175(2): 272 277. 
[2] ARAÚJO J C, SADER M S, MOREIRA E L, et al. Maximum substitution of magnesium for calcium sites in Mg-TCP structure determined by X-ray powder diffraction with the Rietveld refinement. Materials Chemistry and Physics, 2009, 118(2): 337-340.

[3] MATSUNAGA K, KUBOTA $\mathrm{T}$, TOYOURA $\mathrm{K}$, et al. First-principles calculations of divalent substitution of $\mathrm{Ca}^{2+}$ in tricalcium phosphates. Acta Biomaterialia, 2015, 23(1): 329-337.

[4] GRIGG A T, MEE M, MALLINSON P M, et al. Cation substitution in $\beta$-tricalcium phosphate investigated using multi-nuclear, solid-state NMR. Journal of Solid State Chemistry, 2014, 212: 227-236.

[5] NABIYOUNI M, REN Y, BHADURI S B. Magnesium substitution in the structure of orthopedic nanoparticles: a comparison between amorphous magnesium phosphates, calcium magnesium phosphates, and hydroxyapatites. Materials Science and Engineering $C$, 2015, 52: 11-17.

[6] HABRAKEN W, HABIBOVIC P, EPPLE M, et al. Calcium phosphates in biomedical applications: materials for the future? Materials Today, 2016, 19(2): 70-87.

[7] QIN S, LU A H, WANG C Q. The minerals in the human body. Earth Science Frontiers, 2008, 15(6): 32-39.

[8] XIE X D, CHEN M. Formation conditions of tuite. Geochimica, 2008, 37(4): 297-303.

[9] JANG H L, JIN K, LEE J, et al. Revisiting whitlockite, the second most abundant biomineral in bone: nanocrystal synthesis in physiologically relevant conditions and biocompatibility evaluation. Acs Nano, 2014, 8(1): 634-641.

[10] KIM H D, JANG H L, AHN HY, et al. Biomimetic whitlockite inorganic nanoparticles-mediated in situ remodeling and rapid bone regeneration. Biomaterials, 2017, 112: 31-43.
[11] ZHANG J T, LIU W Z, SCHNITZLER V, et al. Calcium phosphate cements for bone substitution: chemistry, handling and mechanical properties. Acta Biomaterialia, 2013, 10(3): 1035-1049.

[12] FURUZONO T, WALSH D, YASUDA S, et al. Preparation of plated $\beta$-tricalcium phosphate containing hydroxyapatite for use in bonded inorganic-organic composites. Journal of Materials Science, 2005, 40(9): 2595-2597.

[13] SWAIN S K, GOTMAN I, UNGER R, et al. Microstructure, mechanical characteristics and cell compatibility of $\beta$-tricalcium phosphate reinforced with biodegradable Fe-Mg metal phase. Journal of the Mechanical Behavior of Biomedical Materials, 2016, 53: 434-444.

[14] SHAVANDI A, BEKHIT E D A, ALI A, et al. Microwave-assisted synthesis of high purity $\beta$-tricalcium phosphate crystalline powder from the waste of green mussel shells. Powder Technology, 2015, 273: 33-39.

[15] TORRES P M, GOUVEIA S, OLHERO S, et al. Injectability of calcium phosphate pastes: effects of particle size and state of aggregation of $\beta$-tricalcium phosphate powders. Acta Biomaterialia, 2015, 21: 204-216.

[16] HASHIMOTO K, KOBAYASHI T, TODA Y. Chemical composition of synthetic whitlockite prepared by hydrothermal method. Inorganic Materials, 1999, 6(279): 110-116.

[17] GOPAL R, CALVO C, ITO J, et al. Crystal structure of synthetic Mg-whitlockite, $\mathrm{Ca}_{18} \mathrm{Mg}_{2} \mathrm{H}_{2}\left(\mathrm{PO}_{4}\right)_{14}$. Canadian Journal of Chemistry, 2011, 52(7): 1155-1164.

[18] BOANINI E, GAZZANO M, BIGI A. Ionic substitutions in calcium phosphates synthesized at low temperature. Acta Biomaterialia, 2010, 6: 1882-1894. 